Enferm Bras 2021;20(1):38-52

doi: 10.33233/eb.v20i1.4263

\title{
ARTIGO ORIGINAL \\ Conhecimento sobre angiografia e níveis de ansiedade em pacientes no período pré-cateterismo
}

Márcio Vitor da Costa Carneiro*, Felipe Carvalho Farias**, Bruno Albuquerque Campos $^{\star * *}$, Tarcísia Domingos de Araújo Sousa****, Fernanda da Mata Vasconcelos Silva ${ }^{\star \star \star * *}$, Emanuela Batista Ferreira e Pereira, D.Sc. ${ }^{\star \star \star \star *}$

*Enfermeiro, Especialista em diagnóstico Universidade de Pernambuco/UPE, Recife, PE, ${ }^{\star \star}$ Enfermeiro, Especialista em Emergência Geral, Universidade de Pernambuco, UPE, Recife,PE, ${ }^{* *}$ Enfermeiro, Mestrando em Perícias Forenses (UPE/FOP), Recife, PE, ${ }^{* * * \star E n f e r m e i r a, ~ M e s t r a n d a ~ e m ~ E n f e r m a g e m, ~}$ Universidade de Pernambuco, UPE, Recife, PE, ${ }^{* * * *}$ Enfermeira, Doutoranda em Enfermagem, Universidade de Pernambuco/UPE, Recife, PE, ${ }^{\star * * * \star}$ Enfermeira, Doutora em Cirurgia pela Universidade Federal de Pernambuco/UFPE, Recife, $P E$

Recebido em 12 de julho de 2020; aceito em 18 de fevereiro de 2021.

Correspondência: Fernanda da Mata Vasconcelos Silva, Rua Vicente do Rego Monteiro,292 Cordeiro 50630-710 Recife PE

\footnotetext{
Márcio Vitor da Costa Carneiro: marciovictordodo@hotmail.com Felipe Carvalho Farias: felipecarvalhof@gmail.com Bruno Albuquerque Campos: bruno.campos@upe.br Tarcisia Domingos de Araújo Sousa: tarcisiadsousa@gmail.com Fernanda da Mata Vasconcelos Silva: nandadamata34@gmail.com Emanuela Batista Ferreira e Pereira: emanuela.pereira@upe.br
}

\section{Resumo}

Objetivo: Analisar o conhecimento sobre angiografia e sua associação com níveis de ansiedade em pacientes no período pré-cateterismo. Métodos: Estudo transversal, descritivo com abordagem quantitativa, realizado no setor de 
hemodinâmica de um hospital de alta complexidade com referência neurocirúrgica e cirurgia vascular. A coleta se deu a partir de um formulário adaptado de um instrumento validado e a aplicação do Inventário de Ansiedade. Os dados foram analisados descritivamente e para avaliar associação entre duas variáveis categóricas foi utilizado o teste Exato de Fisher. Resultados: A faixa etária variou entre 18-86 anos. As comorbidades mais frequentes foram hipertensão arterial sistêmica (48,6\%), diabetes mellitus (24,3\%), insuficiência renal aguda e insuficiência renal crônica com percentuais de 8,1\% e 5,4\% respectivamente. A maioria estava realizando o procedimento pela primeira vez e para diagnóstico. Relataram que as informações sobre o procedimento não foram totalmente esclarecedoras. Quanto ao nível de ansiedade, constatou-se que os níveis presentes foram leves ou moderados e que não houve associações significativas entre o inventário de ansiedade e os resultados das questões sobre as informações prestadas. Conclusão: Faz-se necessário uma comunicação efetiva entre os profissionais, pacientes e familiares, uma vez que foi demonstrado que a qualidade das informações prestadas pode minimizar os níveis de ansiedade.

Palavras-chave: angiografia; ansiedade; doenças cardiovasculares; enfermagem perioperatória; hemodinâmica.

\section{Abstract \\ Knowledge about coronary angiography and anxiety levels in pre- catheterization patients}

Objective: To analyze the knowledge about angiography and its association with anxiety levels in patients in the pre-catheterization period. Methods: Crosssectional, descriptive study with a quantitative approach, carried out in the hemodynamics sector of a highly complex hospital with neurosurgical reference and vascular surgery. The collection took place from a form adapted from a validated instrument and the application of the Anxiety Inventory. The data were analyzed descriptively, and Fisher's Exact test was used to assess the association between two categorical variables. Results: The age range varied between 18-86 years. The most frequent comorbidities were systemic arterial hypertension (48.6\%), diabetes mellitus (24.3\%), acute renal insufficiency and chronic renal insufficiency with percentages of $8.1 \%$ and $5.4 \%$ respectively. Most 
were performing the procedure for the first time and for diagnosis. They reported that the information about the procedure was not entirely clarifying. As for the level of anxiety, it was found that the levels present were mild or moderate and that there was no significant association between the anxiety inventory and the results of the questions about the information provided. Conclusion: Effective communication between professionals, patients and family members is necessary, since it has been demonstrated that the quality of the information provided can minimize the levels of anxiety.

Keywords: angiography; anxiety; cardiovascular diseases; perioperative nursing; hemodynamics.

\section{Resumen}

Conocimiento sobre angiografía coronaria y niveles de ansiedad en pacientes pre-cateterismo

Objetivo: Analizar los conocimientos sobre angiografía y su asociación con los niveles de ansiedad en pacientes en el período de precateterismo. Métodos: Estudio descriptivo transversal con abordaje cuantitativo, realizado en el sector hemodinámico de un hospital de alta complejidad con referencia neuroquirúrgica y cirugía vascular. La recolección se realizó a partir de un formulario adaptado de un instrumento validado y la aplicación del Inventario de Ansiedad (BAI). Los datos se analizaron de forma descriptiva y se utilizó la prueba exacta de Fisher para evaluar la asociación entre dos variables categóricas. Resultados: El rango de edad varió entre 18 y 86 años. Las comorbilidades más frecuentes fueron hipertensión arterial sistémica $(48,6 \%)$, diabetes mellitus $(24,3 \%)$, insuficiencia renal aguda e insuficiencia renal crónica con porcentajes del $8,1 \%$ y $5,4 \%$ respectivamente. La mayoría realizaba el procedimiento por primera vez y con fines de diagnóstico. Informaron que la información sobre el procedimiento no era del todo esclarecedor. En cuanto al nivel de ansiedad, se encontró que los niveles presentes eran leves o moderados y que no existía asociación significativa entre el inventario de ansiedad y los resultados de las preguntas sobre la información brindada. Conclusión: Es necesaria una comunicación efectiva entre profesionales, pacientes y familiares, ya que se ha demostrado que la calidad de la información brindada puede minimizar los niveles de ansiedad. 
Palabras-clave: angiografía; ansiedad; enfermedades cardiovasculares; enfermería peroperatoria; hemodinámica.

\section{Introdução}

As doenças cardiovasculares (DCV) são consideradas distúrbios que acometem o sistema circulatório alterando hemodinamicamente veias, artérias e vasos capilares [1]. Classificadas em: doenças coronarianas, cerebrovasculares, arterial periférica, cardíaca reumática, congênita, trombose venosa profunda e embolia pulmonar [2]. As DCV se apresentam como principal causa de óbito no mundo, atingindo mais de 17,5 milhões de pessoas no ano de 2012, somando mais de $31 \%$ entre todas as causas de morte [2]. No Brasil não é diferente, pois esses índices computam mais de 30\% dessas causas [3].

Assim, observa-se que a incidência das cardiopatias vem aumentando consideravelmente no Brasil e no mundo e, concomitante a este evento, a angiografia por cateteres é considerada como a técnica diagnóstica invasiva mais relevante na prática clínica para os pacientes com distúrbios cardiovasculares [4,5].

A angiografia por cateteres consiste na introdução de um cateter por punção percutânea, que irá seguir um caminho dos vasos sanguíneos da região de punção (região inguinal, periférica ou do braço) até a área de investigação diagnóstica. O cateter, fio flexível, vai sendo guiado por um sistema de Raio-X e o procedimento acontece sob indução anestésica. $\mathrm{Na}$ área de investigação ocorre à infusão de solução de contraste iodado, com a finalidade de identificar obstruções, defeitos congênitos ou estruturais [6].

As manifestações emocionais que ocorrem em pacientes submetidos à angiografia por cateteres decorrem da falta de conhecimento suficiente sobre 0 exame, no qual resulta em situações de apreensão e ansiedade. Os sintomas relacionados ao estresse dificultam o processo de compreensão das informações recebidas por profissionais da saúde acerca do procedimento. [7]. Dessa forma, este estudo objetiva analisar o conhecimento sobre angiografia e sua associação com níveis de ansiedade em pacientes no período précateterismo. 
Material e métodos

Trata-se de estudo transversal, descritivo com abordagem quantitativa, pois foi realizado no período que avaliou de forma simultânea causa e efeito, com a distribuição descritiva dos dados coletados [8,9]. O estudo ocorreu no setor de hemodinâmica de um hospital público de alta complexidade, de referência neurocirúrgica e cirurgia vascular, com 06 montados para este fim. 0 hospital conta com um total de 706 leitos registrados pelo Ministério da Saúde para atendimentos dos pacientes, localizado no município de Recife/PE.

A população foi composta por pacientes que seriam submetidos à angiografia por cateteres, maiores de 18 anos, de ambos os sexos. Amostra nãoprobabilística por conveniência atendeu aos critérios de elegibilidade e foi composta por 37 pacientes. Foram excluídos os pacientes com impossibilidade cognitiva para responder à pesquisa.

Para coleta de dados foram utilizados dois instrumentos. O primeiro um formulário para mensurar o conhecimento sobre angioplastia adaptado pelo autor a partir de um instrumento validado no Brasil em 2002 intitulado "Instrumentos de coleta de dados para o período perioperatório de cirurgia cardíaca" [10]. O segundo foi o Inventário de Beck para mensurar os níveis de ansiedade [11].

O instrumento adaptado foi composto pelas variáveis quantitativas como idade e quantidade de procedimentos realizados; e variáveis qualitativas como sexo, religião, escolaridade, profissionais que disponibilizaram informações. Mensurou-se também como as informações foram recebidas pelos pacientes e se foram suficientes para o conhecimento sobre o procedimento a ser realizado. Outros questionamentos foram levantados desde a decisão de realização da angiografia por cateteres até o momento da entrevista [10].

As Escalas Beck são compostas pelo Inventário de Depressão (BDI), Inventário de Ansiedade (BAI), Escala de Desesperança (BHS) e Escala de Ideação Suicida (BSI). Para mensuração dos níveis de ansiedade utilizamos o Inventário de Ansiedade (BAI) que se caracteriza por ser autoaplicável para maiores de 13 anos, validado no Brasil e utiliza 21 perguntas para avaliar os 
níveis de ansiedade do indivíduo. Os atributos relacionados à ansiedade apresentam scores baseados na seguinte pontuação: 0 a 21 ansiedade baixa, 22 a 35 ansiedade moderada e acima de 36 um grau preocupante de ansiedade [11].

Os dados foram analisados descritivamente através de frequências absolutas e relativas para as variáveis categóricas e das medidas: média, desvio padrão e mediana da variável idade. Para avaliar associação entre duas variáveis categóricas foi utilizado o teste Exato de Fisher, uma vez que a condição para utilização do teste Qui-quadrado não foi verificada. A margem de erro utilizada na decisão dos testes estatísticos foi de $5 \%$. Os dados foram digitados por dupla entrada no Microsoft Excel e importados para o IBM SPSS versão 23 para realizar os cálculos estatísticos.

O estudo está de acordo com a Resolução 466/2012 do Conselho Nacional de Saúde que regulamenta a prática de pesquisas humanas, aprovado no Comitê de Ética em Pesquisa do Hospital da Restauração, sob o número do CAAE: 95940318.8 .0000 .5198$.

\section{Resultados}

$\mathrm{Na}$ caracterização da amostra evidenciou-se que faixa etária dos entrevistados variou entre 18-86 anos, obtendo uma média de 56,22 anos (Desvio padrão: 16,15). Mais da metade era do sexo feminino (56,8\%), 45,9\% concluíram o ensino fundamental e $48,6 \%$ pertenciam a religião católica. As comorbidades estavam presentes em mais da metade dos participantes $(54,1 \%)$, sendo as mais frequentes: hipertensão arterial sistêmica (48,6\%), diabetes mellitus $(24,3 \%)$, insuficiência renal aguda e insuficiência renal crônica com percentuais de $8,1 \%$ e $5,4 \%$ respectivamente.

$\mathrm{Na}$ Tabela I estão dispostas a distribuição das respostas referente às questões sobre o procedimento cirúrgico. Quanto à realização prévia do procedimento $27,0 \%$ dos pacientes informaram já ter se submetido ao cateterismo, e $73,0 \%$ que era a primeira vez e a causa em $73 \%$ das ocorrências foi para fins diagnósticos. O tempo cirúrgico variou em um intervalo de 30 a 50 
minutos $(62,2 \%)$, as principais complicações potenciais elencadas foram dor $(29,7 \%)$ e hemorragias $(18,9 \%)$.

Tabela I - Distribuição das respostas segundo as questões relacionadas ao procedimento e informação. Recife/PE, Brasil, 2019

\begin{tabular}{|c|c|c|}
\hline Variáveis & $\mathrm{N}(37)$ & $\%(100,0)$ \\
\hline \multicolumn{3}{|c|}{ Já realizou procedimento de angiografia por cateteres antes? } \\
\hline $\begin{array}{l}\text { Sim } \\
\text { Não }\end{array}$ & $\begin{array}{l}10 \\
27\end{array}$ & $\begin{array}{l}27,0 \\
73,0\end{array}$ \\
\hline \multicolumn{3}{|c|}{ Número de vezes que realizou o procedimento } \\
\hline Nenhuma & 27 & 73,0 \\
\hline Uma & 04 & 10,8 \\
\hline Duas & 04 & 10,8 \\
\hline Três ou mais & 02 & 5,4 \\
\hline \multicolumn{3}{|c|}{ Motivo da realização da angiografia por cateteres } \\
\hline Diagnóstico & 27 & 73,0 \\
\hline Rotina & 04 & 10,8 \\
\hline Tratamento & 05 & 13,5 \\
\hline Outro & 01 & 2,7 \\
\hline \multicolumn{3}{|l|}{ Tempo de realização do procedimento } \\
\hline 10 minutos & 05 & 13,5 \\
\hline 30 a 50 minutos & 23 & 62,2 \\
\hline Mais de 60 minutos & 09 & 24,3 \\
\hline \multicolumn{3}{|c|}{ Preparos antes da realização do exame (I) } \\
\hline Jejum absoluto & 37 & 100,0 \\
\hline Exame de sangue & 23 & 62,2 \\
\hline Repouso absoluto & 19 & 51,4 \\
\hline Administração de medicação & 11 & 29,7 \\
\hline Suspensão de algumas medicaç̃es & 11 & 29,7 \\
\hline Uso de laxantes & 05 & 13,5 \\
\hline Hidratação oral & 05 & 13,5 \\
\hline Raio-X & 05 & 13,5 \\
\hline Ressonância magnética & 05 & 13,5 \\
\hline Ultrassom & 04 & 10,8 \\
\hline Leves caminhadas & 03 & 8,1 \\
\hline Dieta livre & 01 & 2,7 \\
\hline \multicolumn{3}{|c|}{ Possiveis complicacões do procedimento (1) } \\
\hline & 11 & 29,7 \\
\hline Hemorragia & 07 & 18,9 \\
\hline Cansaço & 05 & 13,5 \\
\hline Alergias & 04 & 10,8 \\
\hline Vômitos & 04 & 10,8 \\
\hline Cefaleia & 03 & 8,1 \\
\hline Risco de morte & 03 & 8,1 \\
\hline Trombose & 02 & 5,4 \\
\hline Edema & 02 & 5,4 \\
\hline Infecção & 01 & 2,7 \\
\hline Outra & 04 & 10,8 \\
\hline
\end{tabular}

${ }^{1}$ Considerando que as variáveis são de múltiplas escolhas a possibilidade da soma das frequências é superior ao total

$\mathrm{Na}$ Tabela II estão descritas respostas referentes às questões sobre 0 primeiro contato assistencial e qualidade das informações disponibilizadas. Os médicos foram os profissionais que tiveram mais contato com o paciente desde a indicação do procedimento (94,6\%), porém 70,3 dos pacientes afirmam que 
tiveram pouca informação sobre o procedimento que seria realizado e 40,5 \% foram transmitidas pelo enfermeiro. As principais informações fornecidas foram sobre indicações para angiografia (78,4\%), importância do exame (35,1\%), contraindicações $(16,2 \%)$ e em relação às etapas do procedimento $(16,2 \%)$. Chama atenção o fato de 45,9\% dos entrevistados relatarem que a qualidade da informação recebida foi "pouco esclarecedora".

Tabela II - Distribuição das respostas segundo as questões relacionadas a informação. Recife/PE, Brasil, 2019

\begin{tabular}{|c|c|c|}
\hline Variáveis & $\mathrm{N}(37)$ & $\%(100,0)$ \\
\hline \multicolumn{3}{|c|}{$\begin{array}{l}\text { Profissionais de saúde que teve contato desde a indicação do procedimento até a realizaçã } \\
\text { mesmo (1) }\end{array}$} \\
\hline Médico & 35 & 94,6 \\
\hline Enfermeiro & 29 & 78,4 \\
\hline Nenhum & 01 & 2,7 \\
\hline Outro & 01 & 2,7 \\
\hline \multicolumn{3}{|c|}{ Foi disponibilizad o algum tipo de informacão sobre o procedimen to realizado? } \\
\hline Muita informação & 06 & 16,2 \\
\hline Pouca informação & 26 & 70,3 \\
\hline Nenhuma informação & 05 & 13.5 \\
\hline \multicolumn{3}{|c|}{ Profissional que disponibilizou as informaç̃es sobre o procedimento (1) } \\
\hline Médico & 25 & 67,6 \\
\hline Enfermeiro & 15 & 40,5 \\
\hline \multicolumn{3}{|c|}{ Informaçỏes que foram disponibilizadas pelo profissional (1) } \\
\hline Tndicaọeses para angiografia & 29 & 78,4 \\
\hline Importância do exame & 13 & 35,1 \\
\hline Contraindicações & 06 & 16,2 \\
\hline Etapas do procedimento & 06 & 16,2 \\
\hline \multicolumn{3}{|c|}{ Ach a que as informaçoes prestad as foram suficientes para compreen der o procedimento? } \\
\hline Totalmente esclarecedor & 01 & 2,7 \\
\hline Esclarecedor & 07 & 18,9 \\
\hline Esclarecedor mas com dúvidas & 07 & 18,9 \\
\hline Pouco esclarecedor & 17 & 45,9 \\
\hline Nada esclarecedor & 05 & 13,5 \\
\hline \multicolumn{3}{|l|}{ Quanto à orientação recebida? } \\
\hline T otalmente orientado & 05 & 13,5 \\
\hline Orientado mas com dúvidas & 09 & 24,3 \\
\hline Pouco orientado & 14 & 37,8 \\
\hline Nada orientado & 09 & 24,3 \\
\hline \multicolumn{3}{|c|}{ Qual/quais dos tópicos a seguir foram abordados durante a anamnese? (1) } \\
\hline Antecedentes pessoais & 29 & 78,4 \\
\hline Alergias & 23 & 62,2 \\
\hline Histórico de AVC/IAM & 21 & 56,8 \\
\hline Doenças cardiovasculares & 20 & 54,1 \\
\hline Doenças pulmonares & 20 & 54,1 \\
\hline Doenças renais & 14 & 37,8 \\
\hline Antecedentes familiares & 12 & 32,4 \\
\hline Sinais de estresse e/ou ansiedade & 07 & 18,9 \\
\hline Nenhum & 02 & 5,4 \\
\hline
\end{tabular}

${ }^{1}$ Considerando que as variáveis são de múltiplas escolhas a possibilidade da soma das frequências é superior ao total

Os resultados do Inventário de Beck encontram-se dispostos na Tabela III, a maioria respondeu nunca ter tido: "Desmaio/cabeça leve" (94,6\%), 
"Dormência ou formigamento" (83,8\%), "Suores quentes/frios" (78,4\%), "Sentindo-se quente" (75,7\%), "Fraqueza nas pernas" (73,0\%), “Indigestão" (73,0\%), "Tontura" (70,3\%), "Mãos tremulas" (70,3\%) e as questões que tiveram os menores percentuais foram "Nervoso" (24,3\%), "Aterrorizado com medo" (35,1\%), "Incapaz de relaxar" (35,1\%), "Instável” (37,8\%), “Assustado” (43,2\%) e "Medo do pior acontecer" (43,2\%).

Tabela III - Distribuição dos pacientes analisados segundo as questões do Inventário de Beck. Recife/PE, Brasil, 2019

\begin{tabular}{|c|c|c|c|c|c|c|c|c|}
\hline & \multicolumn{2}{|c|}{ Nenhum } & \multicolumn{2}{|c|}{$\begin{array}{l}\text { Suavemente, } \\
\text { náo incomodou } \\
\text { muito }\end{array}$} & \multicolumn{2}{|c|}{$\begin{array}{l}\text { Suavemente, } \\
\text { mas incomodou } \\
\text { muito }\end{array}$} & \multicolumn{2}{|c|}{$\begin{array}{l}\text { Severamente, } \\
\text { in comodou } \\
\text { muito }\end{array}$} \\
\hline & $\mathrm{N}$ & $\%(1)$ & $\mathrm{N}$ & $\%(1)$ & $\mathrm{N}$ & $\%(1)$ & $\mathrm{N}$ & $\%(1)$ \\
\hline Dormência & & & & & & & & \\
\hline formigamento & 31 & 83,8 & 03 & 8,1 & 02 & 5,4 & 01 & 2,7 \\
\hline Sentindo-se quente & 28 & 75,7 & 05 & 13,5 & 03 & 8,1 & 01 & 2,7 \\
\hline Fraqueza nas pernas & 27 & 73,0 & 03 & 8,1 & 06 & 16,2 & 01 & 2,7 \\
\hline Incapaz de relaxar & 13 & 35,1 & 10 & 27,0 & 09 & 24,3 & 05 & 13,5 \\
\hline Medo do pior ou acontecer & 16 & 43,2 & 07 & 18,9 & 09 & 24,3 & 05 & 13,5 \\
\hline Tontura & 26 & 70,3 & 05 & 13,5 & 03 & 8,1 & 03 & 8,1 \\
\hline Coração batendo rápido & 21 & 56,8 & 09 & 24,3 & 02 & 5,4 & 05 & 13,5 \\
\hline Aterrorizado ou com medo & 13 & 35,1 & 07 & 18,9 & 13 & 35,1 & 04 & 10,8 \\
\hline Nervoso & 09 & 24,3 & 12 & 32,4 & 09 & 24,3 & 07 & 18,9 \\
\hline Sensaç̃̃o de asfixia & 25 & 67,6 & 02 & 5,4 & 04 & 10,8 & 06 & 16,2 \\
\hline Mãos trêmulas & 26 & 70,3 & 03 & 8,1 & 04 & 10,8 & 04 & 10,8 \\
\hline Medo de perder o controle & 24 & 64,9 & 07 & 18,9 & 03 & 8,1 & 03 & 8,1 \\
\hline Instável & 14 & 37,8 & 07 & 18,9 & 10 & 27,0 & 06 & 16,2 \\
\hline Dificuldade para respirar & 25 & 67,6 & 06 & 16,2 & 03 & 8,1 & 03 & 8,1 \\
\hline Medo de morrer & 19 & 51,4 & 04 & 10,8 & 07 & 18,9 & 07 & 18,9 \\
\hline Assustado & 16 & 43,2 & 09 & 24,3 & 07 & 18,9 & 05 & 13,5 \\
\hline Indigestão & 27 & 73,0 & 05 & 13,5 & 04 & 10,8 & 01 & 2,7 \\
\hline Desmaio/Cabeça leve & 35 & 94,6 & 01 & 2,7 & 01 & 2,7 & - & - \\
\hline Suores quentes/frios & 29 & 78,4 & 01 & 2,7 & 07 & 18,9 & - & - \\
\hline Rosto entusiasmado & 24 & 64,9 & 08 & 21,6 & 05 & 13,5 & - & - \\
\hline
\end{tabular}

${ }^{1}$ Os valores percentuais foram obtidos do número total de 37 pacientes analisados.

Verificou que a maioria $(75,7 \%)$ dos pacientes foi classificada com ansiedade leve, seguido de $21,6 \%$ com ansiedade moderada e apenas um (2,7\%) paciente com ansiedade preocupante. Não foram registradas associações significativas $(p>0,05)$ entre o inventario de Beck e quanto à informação prestada sobre o procedimento e quanto à orientação recebida para o entendimento do procedimento. 
Discussão

A angiografia por cateteres tem cunho diagnóstico e/ou intervencionista, reservada para situações em que os métodos não invasivos, como doppler, angio-tomografia e angio-ressonância são inconclusivas ou dois desses exames são discordantes, entretanto é o método padrão-ouro para o diagnóstico de aneurismas cerebrais nas hemorragias subaracnóideas (HSA) não traumáticas e por conta disso apareceu como maior indicação clínica ao procedimento no estudo $[12,13]$.

A faixa etária idosa encontrada na presente pesquisa diverge da literatura etária, que evidenciaram pacientes com aproximadamente 54 anos e 55,9 anos $[14,15]$. Os achados em relação ao sexo e escolaridade corroboram um estudo coreano, que teve $65,7 \%$ de sua amostra do sexo feminino e $62,8 \%$ dos pacientes possuíam escolaridade menor ou igual ao ensino médio [14].

A frequência de realização de angiografia por cateteres é diferente, quando se compara angiografia cerebral e angiografia cardíaca, pois, um estudo similar evidenciou [16], em sua amostra, que $81,8 \%$ tinham se submetido ao procedimento de angiografia cardíaca anteriormente.

Os pacientes foram submetidos ao procedimento de angiografia por cateteres com o objetivo diagnóstico e uma pequena parcela para tratamento. A pesquisa realizada em uma clínica de neurointervenção coreana identificou que $59 \%$ das angiografias foram realizadas para tratamento e $41 \%$ para diagnóstico [17]. Entretanto, o estudo realizado no Rio Grande do Sul evidenciou que 62\% das angiografias cerebrais por cateter foram de caráter diagnóstico e $37 \%$ foram para controle angiográfico de patologias tratadas anteriormente, o que pode caracterizar o perfil nacional para esse tipo de procedimento [12].

Para a realização desse tipo de procedimento é necessário, além dos equipamentos específicos, uma equipe multidisciplinar especializada para a assistência adequada [12]. É também preciso, anteriormente ao procedimento, a realização de uma consulta minuciosa, em que seja avaliado todo o quadro clínico do paciente e seja informado os detalhes do procedimento [13].

Em um estudo piloto realizado no Estado de São Paulo todos os pacientes foram informados sobre o procedimento a ser realizado, $80 \%$ desses foram informados pelo seu médico, $94,2 \%$ da amostra se mostrou satisfeita com as 
orientações recebidas e, apenas, $21,8 \%$ relataram ansiedade para 0 procedimento [16].

Os dados encontrados no mesmo estudo piloto [16] diferiram do constatado neste, pois para a amostra avaliada as informações prestadas foram insuficientes para compreender o procedimento, tanto a informação prestada sobre a compreensão do procedimento como a qualidade das informações fornecidas sobre o preparo para o procedimento foram insatisfatórias. O fato das informações serem fornecidas por médicos pode ser justificado, pois a angiografia por cateteres tem a marcação realizada pelos mesmos, sendo que a enfermagem, na maioria dos casos, contata o paciente apenas imediatamente antes do procedimento [18].

Entretanto, o enfermeiro desempenha função primordial na unidade de hemodinâmica, uma vez que ele é o responsável pelo gerenciamento do setor e da equipe, além de atuar como educador do paciente, de modo que ele investiga as principais contraindicações e riscos ao paciente, além de orientá-lo para o período pós-procedimento, de modo a permitir a minimização das complicações $[19,20]$.

Não há um consenso na literatura quanto ao preparo para o procedimento, visto que fica a critério do hospital estabelecer as rotinas. A publicação de protocolos institucionais contribui para o fornecimento de alguns critérios a serem realizados e conferidos para todos os exames de cateterismo do paciente, como coagulograma, glicemia e função renal, existência de alergias, além da importância do repouso e outros procedimentos necessários para a boa ocorrência da cirurgia endovascular [21].

Quanto ao jejum absoluto, também não há um consenso para o intervalo de tempo ideal, sendo que a média foi de aproximadamente 8 às 12 horas no hospital onde se fez a pesquisa. Evidências na literatura demonstram que esse tempo é prejudicial à recuperação do paciente, sendo recomendado jejum de $6 \mathrm{~h}$ para uma dieta leve e de $2 \mathrm{~h}$ para líquidos claros, pois há diminuição do estresse sofrido pelo corpo e suas consequências [22].

As complicações do procedimento mais prevalentes foram "dor" e "hemorragia", semelhantes aos encontrados em casos de cateterismos cardiológicos, em que as principais complicações relatadas pelos pacientes são dor lombar, mal-estar geral e dor no local da punção, diferindo apenas quanto à 
hemorragia, pois foi uma complicação bem menos frequente [23]. Assim, uma boa anamnese é imprescindível, pois fornece as informações úteis para a minimização dos riscos e complicações do procedimento, além de fazerem parte do processo de enfermagem e de educação do paciente $[13,20]$.

Os níveis de ansiedade e estresse no período que antecede um procedimento evidenciam a necessidade do profissional da enfermagem em lidar com esse fato, como exemplo da utilização de estratégias de coping, do suporte ao paciente em seus diversos aspectos tais como: religioso, social e familiar [24].

Os dados relacionados aos níveis de ansiedade se assemelham ao de um estudo que avaliou a ansiedade nos pacientes que iriam realizar cateterismo cardíaco, o qual mostrou que os pacientes, mesmo orientados, quanto ao procedimento, apresentaram, em sua maioria sinais de ansiedade leve (entre 30 e $40 \%$ ) e moderada (entre 30 e $36,7 \%$ ). A ansiedade é fator determinante para a ocorrência de doenças cardiovasculares. E quando se relaciona ao procedimento cirúrgico endo vascular, o que mais proporcionou ansiedade aos pacientes foi à possibilidade de insucesso do procedimento e o medo de morrer [25].

Este estudo não registrou associações significativas entre a classificação da ansiedade e o Inventário de Ansiedade, diferindo do que ocorre no préoperatório de cirurgias cardíacas, em que a ansiedade pode ser influenciada, mesmo que de forma mínima, pelas condições demográficas do paciente [26], e os resultados de cada uma das questões sobre as informações prestadas, contrariamente ao constatado por Secco et al. [25], em que comprovou que intervenções educativas são capazes de diminuir os níveis de ansiedade e estresse no pré-procedimento.

Umas das lacunas evidenciadas no estudo era a rotatividade do paciente no leito. O déficit no dimensionamento correto de enfermeiros nos setores da unidade hospitalar contribuiu para assimetria da comunicação entre profissional - paciente - família. A coleta de dados acontecia através da leitura dos prontuários, que por muitas vezes estavam incompletos. Alguns pacientes apresentam baixo nível de compreensão das falas e déficit cognitivo não conseguindo, dessa forma, explanar sobre exames realizados, sintomatologia ou mesmo a patologia que o acomete. Estas foram situações limitantes do estudo que conduziram a exclusão do paciente da amostra. 
O estudo demonstrou que o conhecimento sobre angiografia coronária em pacientes no pré-cateterismo é muito limitado. As informações transmitidas pela equipe de saúde ao paciente foram consideradas pouco esclarecedoras acerca do procedimento que iria ser realizado. O enfermeiro, que deveria mostrar-se como educador em saúde foi pouco atuante nesse processo, conduzindo pacientes e familiares a desenvolverem níveis diversificados de ansiedade.

Porém, ao utilizarmos o Inventário de Ansiedade (BIA) em pacientes précateterismo foram elencados quadros de ansiedade, em sua maioria, leve a moderado. Dessa forma é imprescindível o desenvolvimento de estratégias educativas em saúde voltadas a pacientes indicados para cirurgias cardíacas, uma vez que o acesso a essa informação pode reduzir os níveis de ansiedade e alterações hemodinâmicas pré-operatórias.

\section{Referências}

1. Boubon M, Miranda N, Moura Vicente A, Rato Q. Sabe como prevenir? Doenças Cardiovasculares. Instituto Nacional de Saúde Doutor Ricardo Jorge. Departamento de Promoção da Saúde e Prevenção de Doenças Não Transmissíveis. Lisboa; 2016. http://hdl.handle.net/10400.18/3447

2. OMS. Organização Mundial de Saúde. Doenças Cardiovasculares. Brasília: OMS; 2016.

https://www.paho.org/bra/index.php?option=com content\&view=article\&id=5253:doenc as-cardiovasculares\&ltemid=1096

3. Charlison FJ, Dieleman J, Sing L, Whiteford HA. Donor financing of global mental health, 1995-2015: an assessment of trends, channels, and alignment with the disease burden. Plos One 2017;12(2):e0172259. https://doi.org/10.1371/journal.pone.0172259

4. Oliveira AA, Viana CP, Silva EPB, Maia JS, Pereira MJB, Venturi V, Maia LFS. O cateterismo cardíaco e a enfermagem: a importância dos diagnósticos de enfermagem para uma assistência de qualidade. Revista Científica de Enfermagem 2018;8(23):32142. https://doi.org/10.24276/rerecien2358-3088.2018.8.23.21-27

5. Aguiar BF, Rinaldi ECA Cintho LMM, Martins CLS, Zimmerman MH. Importância dos cuidados de enfermagem no cateterismo cardíaco. Ciênc Cuid Saúde 2016;15(3):460465. https://doi.org/10.4025/cienccuidsaude.v15i3.24894 
6. Sociedade Brasileira de Hemodinâmica e Cardiologia Intervencionista. São Paulo: SBHCl; 2016.

7. Maciel BS, Barros ALBL, Lopes JL. Elaboração e validação de um manual informativo sobre cateterismo cardíaco. Acta Paul Enferm 2016;29(6):633-42. https://doi.org/10.1590/1982-0194201600089

8. Vieira S, Saad HW. Metodologia científica para área de saúde.2ed. Rio de Janeiro: Elsevier; 2015.

9. Polit DF, Beck CT. Fundamentos da pesquisa em enfermagem: avaliação de evidências para a prática de enfermagem. $7^{\mathrm{a}}$ ed. Porto Alegre: ArtMed; 2011.

10. Galdeano LE, Rossi LA. (2002). Construção e validação de instrumentos de coleta de dados para o período perioperatório de cirurgia cardíaca. Revista Latino-Am Enferm 2002;10(6):800-4. https://doi.org/10.1590/S0104-11692002000600008

11. Gomes-Oliveira MH, Gorenstein C, Lotufo Neto F, Andrade LH, Wang YP. Validação da versão brasileira em português do Inventário de Beck-II numa amostra da comunidade. Rev Bras Psiquiatr 2012;34(4):389-94. https://doi.org/10.1016/j.rbp.2012.03.005

12. Vanzin, J, Azambuja Junior ND, Frighetto L, Frohlich AC, Varela DL, Mesquita Filho $P$ et al. Endovascular neurosurgery in the northern macro-region of Rio Grande do Sul: Part I. Arquivos Brasileiros de Neurocirurgia: Brazilian Neurosurgery 2016;37(03):16773. https://doi.org/10.1055/s-0036-1584685

13. Rodrigues MGJ, Silva R., Dias MG, Fernandes AP. Processo de enfermagem em pacientes submetidos à angioplastia transluminal percutânea coronária. Revista Eletrônica Acervo Saúde 2019;1:(23), e284. https://doi.org/10.25248/reas.e284.2019

14. Kwon MA, Hong C-K, Joo J-Y, Kim YB, Chung J. Headache after cerebral angiography: frequency, predisposing factors, and predictors of recovery. J Neuroimaging 2015;26(1):89-94. https://doi.org/10.1111/jon.12290

15. Zanettini A, Silva JC, Boeno MG, Carretta MB. Complicações em pacientes submetidos à intervenção coronária percutânea. REPENF - Rev Parana Enferm 2020;3(1):38-45. http://seer.fafiman.br/index.php/REPEN/article/view/600

16. Silva MAS, Marques PTV, Castro DFA, Padula MPC, Yano KT, Coimbra ALL, et al. Relação entre orientação, ansiedade e dor em pacientes submetidos ao cateterismo cardíaco. Arq Med Hosp Fac Cienc Med Santa Casa São Paulo 2016;61:28-34. https://pdfs.semanticscholar.org/b035/133cfc94a246843a4b11711070ab002d04ab9.pdf

17. Liu H, Park D, Hwang SM, Lee GY, Lim OK, Kim M et al. Outpatient day-care neuroangiography and neurointerventionof unruptured intracranial aneurysms. Neurointervention 2016;11(1):37-41. https://doi.org/10.5469/neuroint.2016.11.1.37

18. Botura AGC, Nishimura BT, Florêncio LDB, Guimarães SML. Relação entre ecocardiografia e cineangiocoronariografia em pacientes diagnosticados com síndrome coronariana aguda. Estudo retrospectivo. Revista da Sociedade Brasileira de Clínica Médica 2017;15(1):6-10. http://www.sbcm.org.br/ojs3/index.php/rsbcm/article/view/240/228 
19. Capetini AC, Camacho ACLF. Nursing care in the hemodynamics service in interventional cardiology: Integrative Review. Research, Society and Development 2020;9(7):e284974200. https://doi.org/10.33448/rsd-v9i7.4200

20. Bruggmann MS, Machado CN, Mazera MS, Schneider DG, Pires PDE. Atuação da enfermagem no serviço de radioterapia (1998-2018). Archives of Health 2020;1(5):16377. https://doi.org/10.46919/archv1n5-001

21. Assunção M, Oliveira APL. Manual de normas e rotinas de procedimentos endovasculares extracardíacos do serviço de hemodinâmica do Hospital Getúlio Vargas. Hospital Getúlio Vargas 2012. http://www.hgv.pi.gov.br/download/201208/HGV24 0d3f1c6437.pdf

22. Campos SBG, Barros-Neto JA, Guedes GS, Moura FA. Pre-operative fasting: why abbreviate? ABCD Arquivos Brasileiros de Cirurgia Digestiva 2018;31(2):e1377-e80. https://doi.org/10.1590/0102-672020180001e1377

23. Castro YTBO, Rolim ILTP, Silva ACO, Silva LDC. Conhecimento e significado do cateterismo cardíaco para pacientes cardiopatas. Rev Rene 2016;17(1):29-35. https://doi.org/10.15253/2175-6783.2016000100005

24. Gonçalves KKN, Silva JI, Gomes ET, Pinheiro LLS, Figueiredo TR, Bezerra SMMS. Ansiedade no período pré-operatório de cirurgia cardíaca. Rev Bras Enferm 2016;69(2):397-403. https://doi.org/10.1590/0034-7167.2016690225i

25. Secco AC, Benincá C, Scortegagna SA, Tognon AP, Espíndola AV, Mognon J. Intervenção psicológica vídeo-orientativa em pacientes submetidos ao cateterismo cardíaco. Psico. 2017;3(48):205-215. https://doi.org/10.15448//1980$\underline{8623.2017 .3 .22773}$

26. Rodrigues HF, Furuya RK, Dantas RAS, Dessotte CAM. Ansiedade e depressão em cirurgia cardíaca: diferenças entre sexo e faixa etária. Esc Anna Nery 2016;20(3):e20160072. https://doi.org/10.5935/1414-8145.20160072 